\title{
Concept study of an implantable microsystem for electrical resistance and temperature measurements in dairy cows, suitable for estrus detection
}

\author{
Raul Morais ${ }^{\mathrm{a}, *}$, A. Valente ${ }^{\mathrm{a}}$, J.C. Almeida ${ }^{\mathrm{b}}$, Amélia M. Silva ${ }^{\mathrm{a}}$, Salviano Soares ${ }^{\mathrm{a}}$, \\ M.J.C.S. Reis ${ }^{\mathrm{a}}$, R. Valentim ${ }^{\mathrm{c}}$, Jorge Azevedo ${ }^{\mathrm{b}}$ \\ ${ }^{a}$ CETAV, Centro de Estudos Tecnológicos, do Ambiente e da Vida, UTAD University, Quinta de Prados, Apartado 1013, 5001-801 Vila Real, Portugal \\ ${ }^{\mathrm{b}}$ CECAV, Centro de Ciência Animal e Veterinária, UTAD University, Quinta de Prados, Apartado 1013, 5001-801 Vila Real, Portugal \\ c IPB, Instituto Politécnico de Bragança, Campus de Santa Apolónia, Apartado 1138, 5301-854 Bragança, Portugal
}

Received 9 September 2005; received in revised form 13 March 2006; accepted 11 April 2006

Available online 5 June 2006

\begin{abstract}
In cattle breeding industry, where artificial insemination techniques are employed, the successful detection of estrus onset leads to considerable cost-saving in herd management. One of the most reliable approaches is based on the determination of progesterone concentration in milk. However, these methods rely on the biosensor concept where a biological substrate is used in a chemical-binding reaction to directly or indirectly produce some effect (electrical or light) that is used at the transducer level. These methods present several drawbacks concerning real-time measurements due to the complexity of the reactions involved and reagent/waste handling. Another approach is to combine measurements of temperature and electrical resistance of reproductive tissues to predict estrus. Using a low-power microsystem with wireless capabilities it is possible to take these measurements in situ and more frequently. The proposed microsystem comprises a second-order delta-sigma modulator for analog-to-digital conversion and a class-E radio-frequency (RF) transmitter operating in the ISM-band of $433 \mathrm{MHz}$ to transfer acquired data to a collar. Electrical resistance is measured by using a modified Wenner array and temperature by the on-chip temperature sensor. System (including battery and antenna) package is made of a tissue-compatible material to allow implantation in the cow's vulvar muscle. Since estrus prediction is based on relative changes of the two correlated parameters, calibration is not necessary. Some preliminary results regarding the measuring concept are presented. (C) 2006 Elsevier B.V. All rights reserved.
\end{abstract}

Keywords: Microsystem; Electrical resistivity sensor; Wireless interface; Estrus prediction

\section{Introduction}

Monitoring progesterone levels in milk is an effective method not only for predicting ovulation, and thus time to artificial insemination, but also for detecting pregnancy and fertility problems. Laboratorial measurements, based on radioimmunoassay (RIA) or enzyme-linked immunosorbent assay (ELISA) methodology, have allowed estrus detection with 98\% specificity, but these methods require time and specific skills [1]. Several approaches have been developed by researchers to determine progesterone in milk and blood [2-4]. Other stud-

\footnotetext{
* Corresponding author. Tel.: +351 259 350343; fax: +351 259350300.

E-mail addresses: rmorais@utad.pt (R. Morais); avalente@utad.pt (A. Valente); jalmeida@utad.pt (J.C. Almeida); amsilva@utad.pt (A.M. Silva); salblues@utad.pt (S. Soares); mcabral@utad.pt (M.J.C.S. Reis); valentim@ipb.pt (R. Valentim); jazevedo@utad.pt (J. Azevedo).
}

ies have shown that electrical impedance in vaginal mucus may be used to pinpoint proper insemination time [5]. Decreasing electrical resistance (ER) values were always associated with the onset of estrus. Nevertheless it should be kept in mind that vulvar ER measurements vary among cows. A suitable solution should monitor the relative changes within cows during the estrus cycle. Once the ER readings begin to decline, the cow should be probed more frequently until the lowest reading is obtained.

Complementary to the ER variation during the estrus cycle, a temperature variation is also present and can be correlated. It has been reported that $12 \mathrm{~h}$ before ovulation the vaginal temperature increase from $37.94 \pm 0.33$ to $39.00 \pm 0.64^{\circ} \mathrm{C}$, as compared to the pre-estrus value [6]. It was also reported that the prediction of estrus based on vaginal temperature was excellent when an increase of at least $0.4{ }^{\circ} \mathrm{C}$ in vagina temperature was observed for 3 or more consecutive hours [7]. 


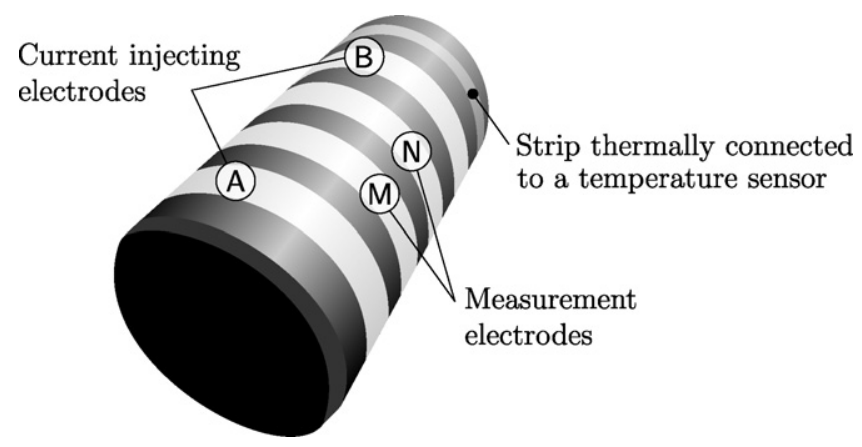

Fig. 1. Perspective of the proposed microsystem package illustrating the modified Wenner array used to measure electrical resistance and temperature.

The combination between these two parameters not only allows a greater specificity in prediction, but enables the conception of a complete and autonomous microsystem for ER and body temperature measurements, suitable to be implanted in the cow vulvar muscle. To achieve this purpose some key issues have been specially addressed: measurement methods, data transfer, power consumption and package (or encapsulation). System conception and measurement methods are presented.

\section{System overview}

The specificity of the proposed methodology dictates that size and type of encapsulation is a major concern. For a reliable and long-term operation, the device must be small, bio-compatible and power-efficient. Regarding energy consumption, data transfer is accomplished by using a highly efficient RF-transmitter operating in class-E mode to transfer small data packets every hour to a receiver device located in the cow collar. Issues concerning data exchange with a collecting and/or processing station are not discussed here. Sensor implementation for measuring ER is based on a four-point electrode arrangement which consists of outer-current injecting electrodes and inner-voltage measurement electrodes with the same separation distance between every electrode. This arrangement is called Wenner alpha type, which has been modified to allow measurements using four conductive strips in the perimeter of the package, as depicted in Fig. 1.

In a Wenner structure, two electrodes (A and B) are used to create a current that flows away from or towards each electrode across the surface. The total potential difference between the electrodes $\mathrm{M}$ and $\mathrm{N}$ is given by [8]:

$V_{\mathrm{MN}}=V_{\mathrm{M}}-V_{\mathrm{N}}=\frac{\rho I}{2 \pi}\left[\left(\frac{1}{\overline{\mathrm{AM}}}-\frac{1}{\overline{\mathrm{MB}}}\right)-\left(\frac{1}{\overline{\mathrm{AN}}}-\frac{1}{\overline{\mathrm{NB}}}\right)\right]$

where $\rho$ represents the surface resistivity $(\Omega \mathrm{m})$ and $I$ is the current (A) that flows between electrodes A and B. Eq. (1) may be rearranged to yield:

$V_{\mathrm{MN}}=\frac{\rho I}{K}$

where

$K \equiv 2 \pi\left[\left(\frac{1}{\overline{\mathrm{AM}}}-\frac{1}{\overline{\mathrm{MB}}}\right)-\left(\frac{1}{\overline{\mathrm{AN}}}-\frac{1}{\overline{\mathrm{NB}}}\right)\right]^{-1}$

is the "geometric factor" that will acquire a particular value for a given electrode spacing. For the Wenner array, all of the separations are equal to a constant value $a$ and the Wenner geometric factor assumes the simple form $K=2 \pi a$. Thus, the apparent resistivity is

$\rho=\left(\frac{V_{\mathrm{MN}}}{I}\right) K$

By knowing the injected current value $I$, ER variations can be accomplished by measuring the potential difference $V_{\mathrm{MN}}$ at the inner electrodes. Temperature variations are detected by using a precision temperature sensor, thermally connected to a metal strip outside the capsule (fifth strip in Fig. 1). Fig. 2 shows a simplified block diagram of the entire system.

The excitation block, basically a sine-step current source, generates an ac current that flows through outer electrodes A and B. The measurement block is responsible for analog multiplexing and for analog-to-digital conversion. The core of this block is a second-order delta-sigma $(\Delta \Sigma)$ modulator, imple-

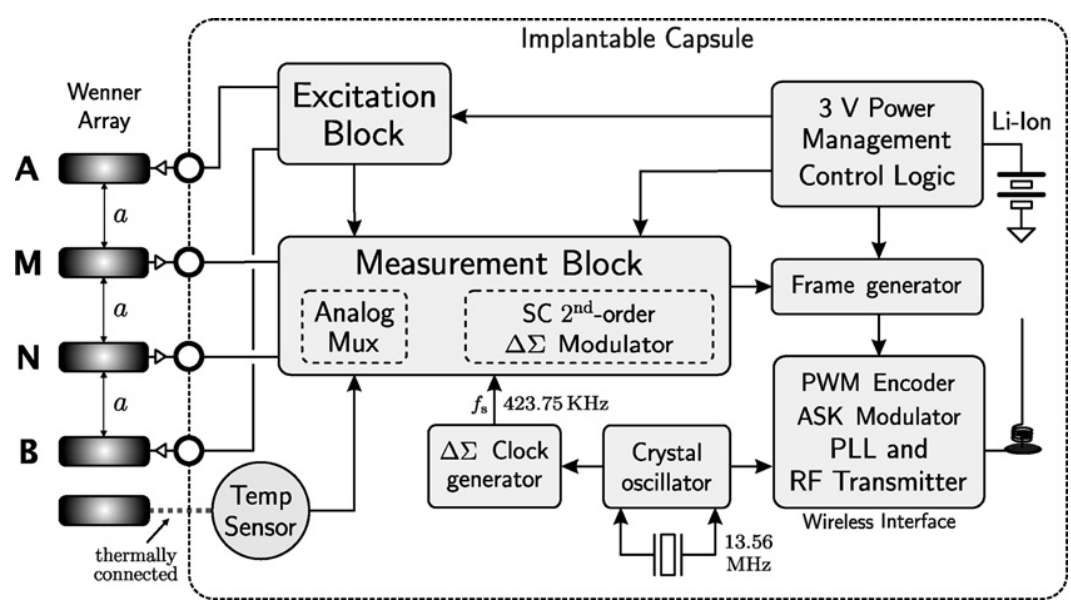

Fig. 2. Block diagram of the proposed microsystem. 
mented in a fully differential topology using switched-capacitor techniques. The output of the $\Delta \Sigma$ modulator (bitstream) is then applied to a first-order digital filter to provide a 14-bit word. The associated control logic is responsible for controlling the analog multiplexer and for digital word synchronization, regarding the temperature sensor and potential difference samples. For data transmission, digital samples are assembled in a frame containing ID, preamble and checksum control fields. Prior to transmission, data is encoded as a pulse-width modulated (PWM) signal and then transmitted, by means of amplitude shift-keying (ASK) modulation, through a power amplifier operating at $433.92 \mathrm{MHz}$ ISM band in class-E mode. The RF frequency is generated by an on-chip frequency synthesizer based on a $13.56 \mathrm{MHz}$ crystal.

The temperature sensor, developed in a previous work [9], is a proportional to absolute temperature (PTAT) circuit with bipolar devices fabricated in the CMOS process. It uses a dynamic PTAT voltage generator with thirty two switching stages using dynamic element matching and dynamic amplification techniques to achieve a high-performance temperature sensor with high accuracy (better than $0.1 \%$ ) and high resolution (better than $0.05^{\circ} \mathrm{C}$, typical $0.01^{\circ} \mathrm{C}$ ).

\section{Electrical bio-impedance}

One of the classic signs of estrus in cows is a swollen vulva. Such swelling is the result of changes in tissue hydration, which causes changes in ER [10]. Changes in cell density, fluid volume, and electrolyte content of bovine vulvar tissue can be accomplished by considering the living tissue as an electrical circuit. The simplified circuit used to simulate electrical properties of a biological subject includes parallel resistance $R_{\mathrm{e}}$ (extracellular space resistance) to serially connected $R_{\mathrm{i}}$ (intracellular space resistance) with $C_{\mathrm{m}}$ (membrane capacitance), as depicted in Fig. 3.

To determine tissue water and composition a bioelectrical impedance analysis (BIA) technique is used. This technique is an assessment of changes in a biological object reflected by the capacitive reactance, resistance, and other factors for an indication of any alteration in the tissue composition.

\section{Measurement methods}

The biological object impedance is measured by injecting a small alternating current, $i_{\text {ext }}(t)$, of constant amplitude at a fixed frequency through the tissue by using two electrodes. The current injection develops a voltage distribution on the periphery of the tissue, related to the impedance distribution of the interior, which is measured using two adjacent electrodes. This voltage drop, $v(t)$, can be seen as the product of $\dot{Z}_{X}$ times $i_{\text {ext }}(t)$, as depicted in Fig. 4. A shift in the phase angle between the injected

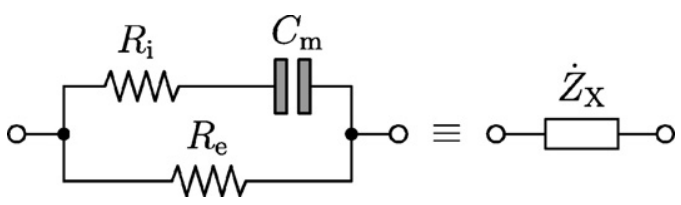

Fig. 3. Equivalent electric circuit for a living tissue.

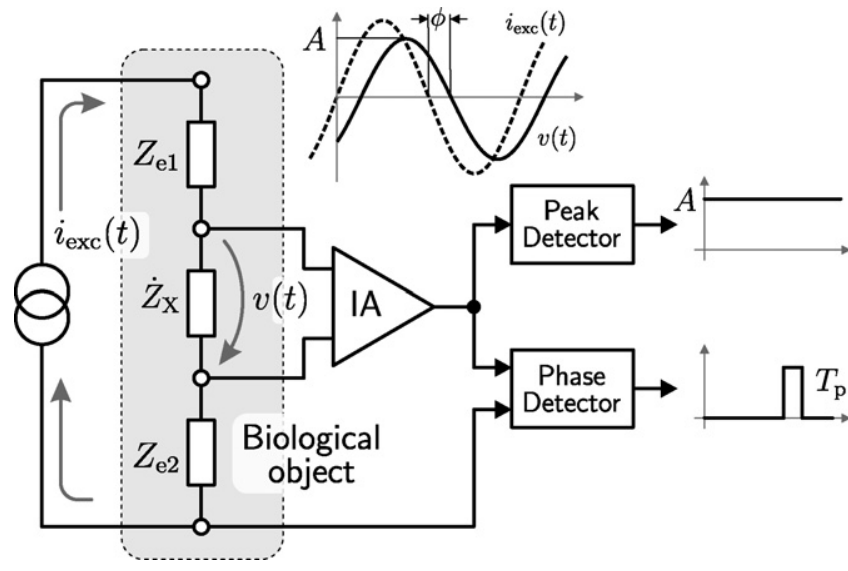

Fig. 4. Principle of operation of an ac impedance measurement system.

current and the measured voltage drop defines reactance or a complex impedance measurement including the dielectric nonconducting space attributed to cell membrane capacitance.

In clinical practice, and for most of the numerous experiments published in the literature [11], the BIA measurements are conduced using a four-electrode, or tetrapolar, method with a fixed frequency of about $50 \mathrm{kHz}$. The reason for this value is that it reduces electrode polarization anomalies and it ideally cancels the influence of the electrode-electrolyte interface impedance [12].

\subsection{Excitation circuit}

To efficiently measure ac impedance, an integrated sinusoidal current source is needed. Most sine current generators are based on a direct digital synthesizer (DDS), which requires a readonly memory (ROM) sine look-up table and a current-mode digital-to-analog converter (DAC). This solution requires a large integration area since waveform generation is closely related to the ROM size and the DAC resolution. Instead of using such approach a set of precisely scaled current mirrors can be used to produce sine steps as depicted in Fig. 5.

Switches $S_{0}$ to $S_{5}$ are turned $O N$ by an up/down counter to produce a half-sine wave, where individual current mirrors $\left(\mathrm{M}_{2,0}, \ldots, \mathrm{M}_{2,5}\right)$ have been calculated to perform an accurate sine current step. Excitation current $i_{\text {ext }}(t)$ (equivalent to a rectified sine waveform) is then mirrored by a wide swing cascade current mirror $\left(\mathrm{M}_{5}, \mathrm{M}_{6}\right)$ and transformed to a full wave by the switching network formed by switches $\mathrm{S}_{\mathrm{A}}, \mathrm{S}_{\mathrm{B}}, \mathrm{S}_{\mathrm{C}}$ and $\mathrm{S}_{\mathrm{D}}$. The reference current $I_{\mathrm{REF}}$ is generated by a bandgap reference circuit and can be adjusted externally during prototype evaluation.

\subsection{Measurement circuit}

Voltage drop at the inner electrodes of the array is characterized by its amplitude (real part of the measured impedance) and the phase shift between the injected current and the measured voltage. Both values must be acquired to calculate the value of the complex impedance $\dot{Z}_{X}$.

The amplitude measurement is based on two peak detectors that detect and hold the minimum and the maximum values of 


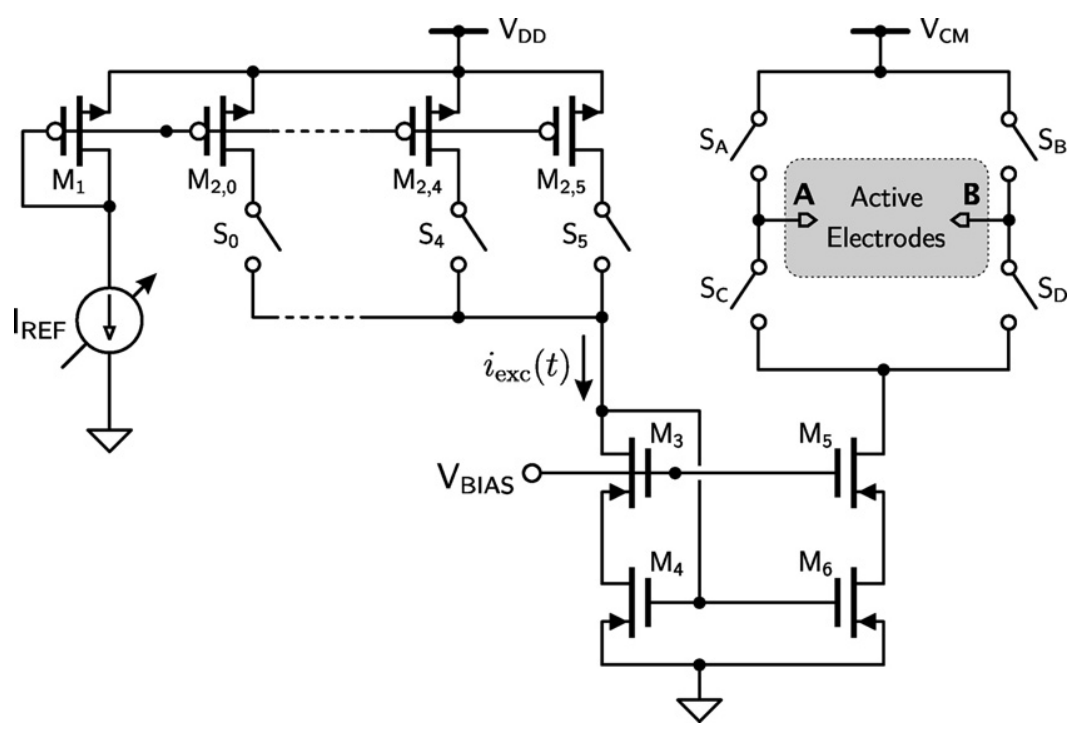

Fig. 5. Sine step current source generation.

the difference between signals at the measuring electrodes, as illustrated in Fig. 6.

Due to the switching action of the excitation circuit, the voltage across $\dot{Z}_{X}$ appears with some induced glitches caused by $\mathrm{S}_{\mathrm{A}}, \mathrm{S}_{\mathrm{B}}, \mathrm{S}_{\mathrm{C}}$ and $\mathrm{S}_{\mathrm{D}}$. Because of this, two first-order gm-C filters are used before the difference amplifier. This type of filter, where gm cells replace resistors, offers several advantages for this application such as low-area, low-power and more accurate integrated filters. The difference amplifier has a fixed gain of five and has been designed using switched-capacitor techniques. The output dc level has been set to the common-mode voltage of the fully differential $\Delta \Sigma$ modulator, allowing both peak detectors to operate within the input dynamic range of the modulator. It should be kept in mind that the use of the minimum peak detector, whose output value is theoretically the opposite of the maximum value, doubles the signal dynamic range and enables the use of fully differential structures. Advantages of using fully differential topologies, such as the case of the $\Delta \Sigma$ modulator, are the reduction of the common-mode interference, switch charge injection and clock feedthrough. Also, and since we are only interested in the amplitude of the measured waveform, the usage of both peak detectors reduces substantially the bandwidth requirements of the $\Delta \Sigma$ modulator.

Phase shift between the applied sine wave and the obtained signal is measured through a phase detector that consists of a differential pair and a comparator, as basically depicted in Fig. 7. The output of the comparator is then applied to a counter clocked by the same clock signal of the $\Delta \Sigma$ modulator to estimate pulsewidth $T_{\mathrm{p}}$.

While one of the inputs is tied to electrodes de level $\left(V_{\mathrm{CM}}\right)$, the other is connected to one of the active electrodes. When opening switch $\mathrm{S}_{\mathrm{A}}$ (see Fig. 5) and closing $\mathrm{S}_{\mathrm{B}}$, the input $V_{1}$ surges over $V_{\mathrm{CM}}$ due to the capacitive effect of the complex impedance. With this circuit, the duration of the pulse produced by the comparator is proportional to the phase shift of the $\dot{Z}_{X}$ impedance. However, and as stated in [13], due to the use of a medium frequency $(50 \mathrm{kHz}$, for example), the capacitive part of the complex impedance can be neglected. Nevertheless, and for future experiments in accessing tissue composition, this block will be integrated as a part of the measuring circuit.

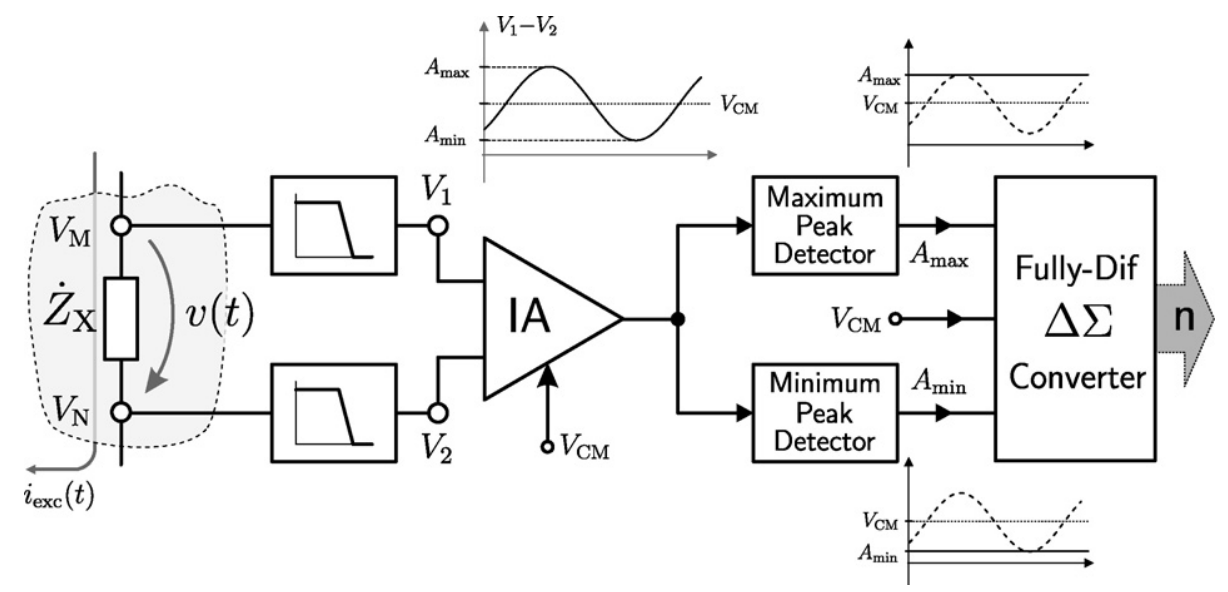

Fig. 6. Amplitude measurement circuit block diagram. 


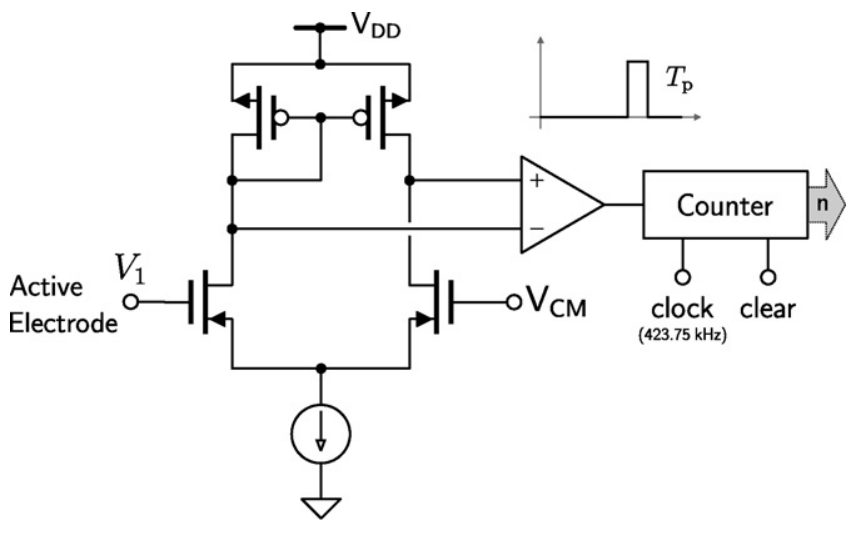

Fig. 7. Phase detector circuit.

\section{Data transfer and encapsulation}

For a feasible operation and continuous surveillance of a cow, the proposed device must remain permanently on the proper anatomical location for the duration of the animal's productive life. One of the requirements dictates that all subsystems must follow low-power and low-voltage design guidelines. In every radio-frequency device, power consumption during transmissions is a major parcel in power budget, even in highly efficient transmitters. To minimize this restriction, as well as power consumption regarding computational power, data is transmitted to a receiver unit located in a cow collar for further processing.

Considering that a receiver is also included, a significant improvement in device lifetime can be achieved since measured data is only needed during the cow estrus cycle. So, data transfer can occur in a dynamic way to reduce power consumption during the times that monitoring is not needed, such as when the cow is pregnant. This is controlled by the receiver unit that issues an interrogation command that wakes up the device, which responds with a data frame and immediately enters in sleep mode. Interrogation commands are issued at a frequency dictated by the cow's estrus cycle starting with a 12-h period and achieving a 1-h period when tracking minimum ER and maximum temperature values. The receiver unit, besides device interrogation function, is also responsible for data exchange within a wireless mesh-topology network, such as $\mathrm{ZigBee}^{\mathrm{TM}}$.

On the other hand, a proper encapsulation must be addressed to ensure that after the device is implanted it presents no discomfort to the animal. To avoid tissue damage or electrode degradation, the electrodes are made with stainless steel (noble metals such as Pt or Au can also be used). The electrodes are located outside the plastic capsule, containing in its interior a Li-ion battery and the antenna. After assembly, the package can be hermetically sealed.

\section{Results}

To fulfill the requirements of a fully autonomous implantable capsule, several subsystems are being designed, simulated and tested. For this prototype, a macrodevice, regarding the measuring method, has been built to validate the proposed methodology.

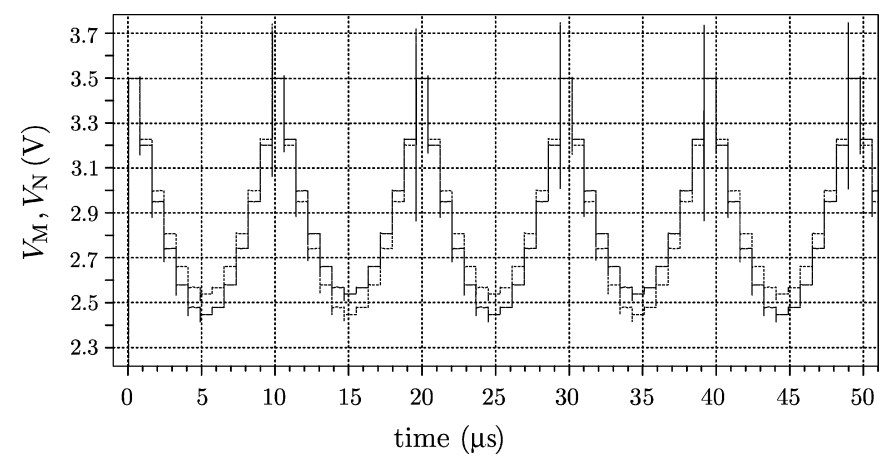

Fig. 8. Simulation of the voltage present at the inner electrodes $\mathrm{M}$ and N, corresponding to a current of $652 \mu \mathrm{A}_{\mathrm{RMS}}$ through electrodes $\mathrm{A}$ and $\mathrm{B}$, assuming three equal resistances of $100 \Omega$ between the four-electrode array.

Simulations were performed in T-Spice from Tanner Tools using the SPICE typical models from the AMI C07M-A CMOS process, available through Europractice program.

The simulated voltages present at the measuring electrodes $\mathrm{M}$ and $\mathrm{N}$ are shown in Fig. 8. This simulation corresponds to an alternating current of approximately $52 \mathrm{kHz}$. Fig. 9 shows the difference between the voltages present at the measuring electrodes, illustrating the sine step current.

Voltages at the measuring electrodes, $V_{\mathrm{M}}$ and $V_{\mathrm{N}}$, are then individually applied to a first-order gm-C filter and the result is applied to the difference amplifier. Fig. 10 shows the simulated response of the two peak detectors fed by the output of the amplifier.

To simulate the behavior of the implantable capsule, some plastic cylinders with the four-electrode array have been built

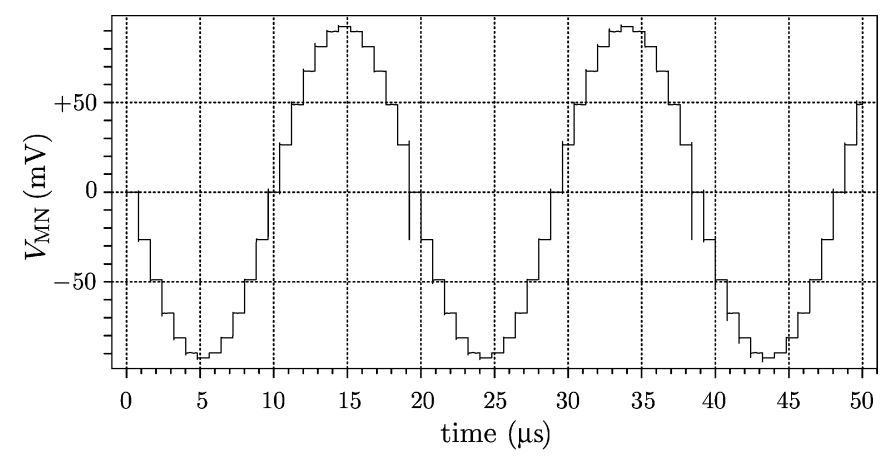

Fig. 9. Simulation of the differential voltage drop at the measuring electrodes.

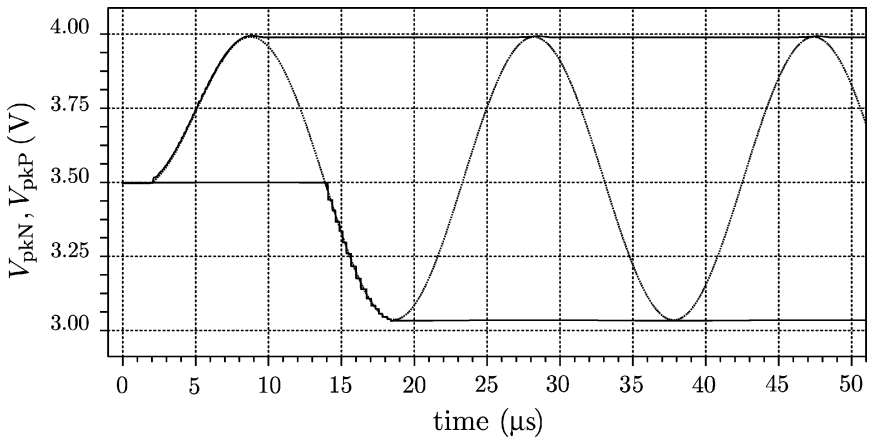

Fig. 10. Simulation of the peak detectors response after filtering. 


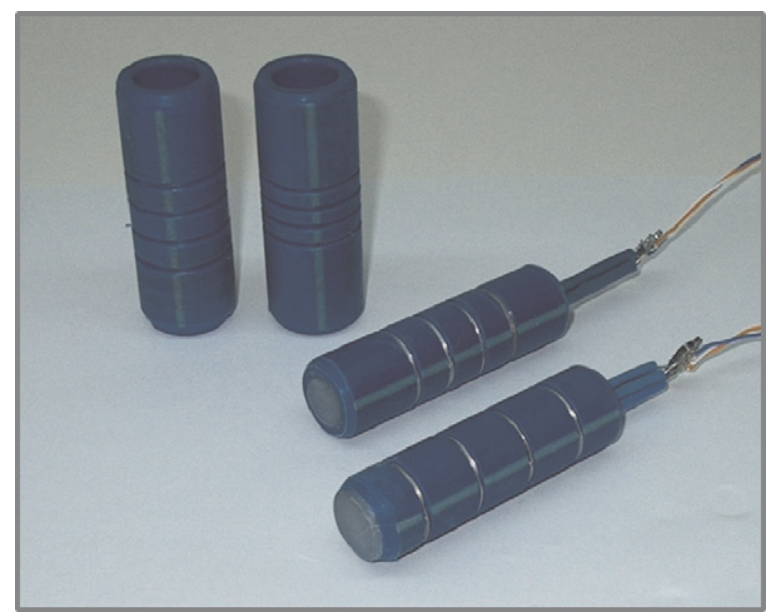

Fig. 11. Several capsule prototypes.

with different electrode separations, as depicted in Fig. 11. The materials used (PVC for the cylinder and stainless steel for the electrodes) were specifically chosen to ensure biocompatibility. In order to keep the electrodes in place, a small chamfer was carved around the external side of the cylinder. All electrical connections were made on the inside of the cylinder, which was then filled with a non-conductive glue. To simulate the electrical conductivity of the vaginal mucus, each capsule was then placed in an aqueous solution of sodium chloride $\left(0.08 \mathrm{~mol} \mathrm{~L}^{-1}\right.$ at $23^{\circ} \mathrm{C}$ ).

At this stage, capsule evaluation was performed by a macrodevice, Fig. 12, where discrete electronic components were employed to generate a sine step current source. This macrodevice, beside the current source, filters and peak detectors, has a 14-bit analog-to-digital converter, a microcontroller and a RS232 driver to acquire and transfer collected data to a laptop computer for posterior analysis. Fig. 13 shows the results of some measurements.

Fig. 13 shows the injected sine wave corresponding to an ac current of amplitude of approximately $660 \mu \mathrm{A}_{\mathrm{RMS}}\left(933 \mu \mathrm{A}_{\text {peak }}\right)$

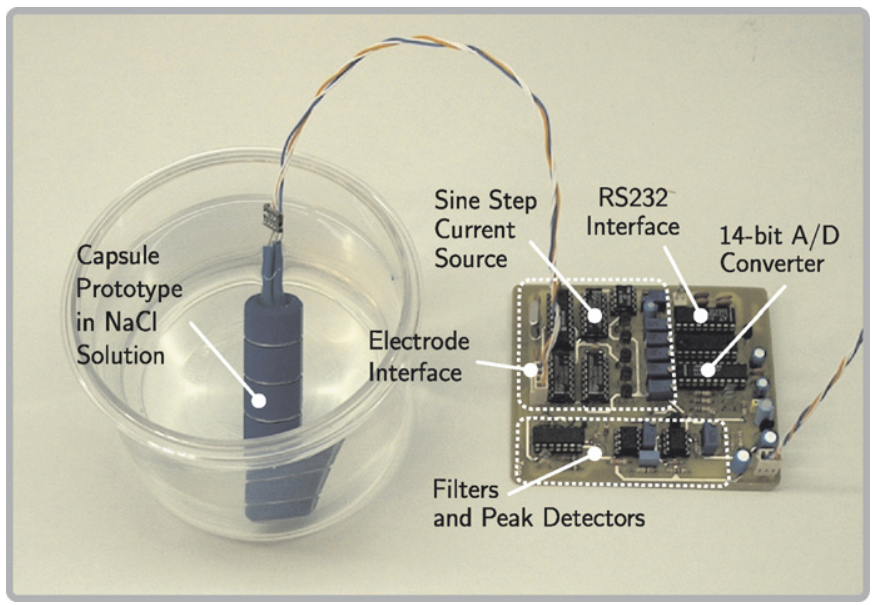

Fig. 12. Experimental setup using a macrodevice to evaluate capsule behavior inside an aqueous solution.

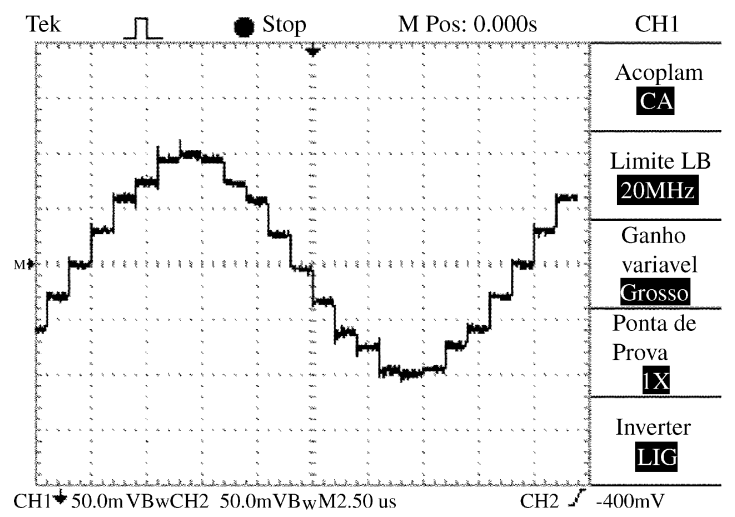

(a)

Injected sine wave

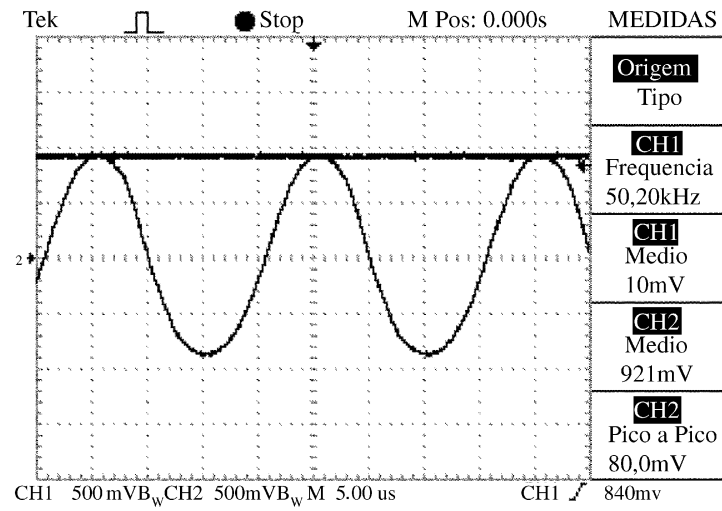

(b)

Peak detector response

Fig. 13. Waveforms obtained using a macrodevice.

and frequency of $52 \mathrm{kHz}$. The waveforms at both outputs of the amplifier and peak detector are shown in Fig. 13 (minimum not shown). The peak detector output indicates a value of $921 \mathrm{mV}$ (gain of the amplifier was set to 10), which is consistent with a $100 \Omega$ resistance measurement. In fact, for a specific capsule with its particular cell constant value, the result is the inverse of the measured conductance, which was $9.8 \mathrm{mS}$. The inverse of the conductance is the resistance leading to a measured value of $102 \Omega$.

\section{Conclusions}

The preliminary results suggests that the proposed implantable microsystem for vulvar ER and body temperature measurements can fulfill the requirements of a low-cost autonomous system to help estrus prediction in herd management.

The major advantage of the presented solution is the capability of continuous monitoring of two correlated parameters in estrus detection during a long period of time without human intervention. A prototype, comprising the excitation and measurement circuits, as well as the RF transmitter, is being implemented in a standard CMOS $0.7 \mu \mathrm{m}$ mixed-signal process (AMIS C07M-A) to validate the proposed methodology. Previous implementations $[14,9]$ have shown that the delta-sigma modulator exhibits an effective 16.1-bit resolution $(98.7 \mathrm{~dB}$ dynamic range) with an $800 \mathrm{~Hz}$ bandwidth. The temperature sen- 
sor has a $0.05{ }^{\circ} \mathrm{C}$ precision, which is suitable to detect very small variations of temperature, which is also closely associated with the onset of standing estrus and the pre-ovulatory surge in Luteinizing hormone (LH).

Another important advantage is related to calibration. Since the proposed capsule will monitor relative changes of temperature and ER during the estrus cycle of different animals, absolute readings are not necessary, thus avoiding calibration of the device.

After fabrication of this prototype, a set of long-term experiments will be scheduled to test the reliability of the capsule, which will be at the proper anatomical location of the animal. During this evaluation phase, the prototype will be scaled down to a $3.3 \mathrm{~V}$ CMOS process (AMIS C035M-A) to allow a single $\mathrm{Li}$-ion cell as power source. Some improvements are also scheduled such as a receiver to control device shutdown and wake-up modes.

To our knowledge, it's the first time that an implantable capsule with a circular strip Wenner array and a microsystem is used to predict estrus in dairy cows. The key of the proposed measurement method is the correlation of the ER and temperature variation, which have been used separately to predict estrus, each one with a reasonable specificity. By combining these two parameters, we not only increase specificity but also the reliability of the prediction.

\section{References}

[1] M.N. Velasco-Garcia, T. Mottram, Biosensor technology addressing agricultural problems, Biosyst. Eng. 84 (2003) 1-12.

[2] R.M. Pemberton, J.P. Hart, T.T. Mottram, An electrochemical immunosensor for milk progesterone using a continuous flow system, Biosens. Bioelectron. 16 (2001) 715-723.

[3] R.W. Claycomb, M.J. Delwiche, Biosensor for on-line measurement of bovine progesterone during milking, Biosens. Bioelectron. 13 (1998) 1173-1180.

[4] Y.F. Xu, M. Velasco-Garcia, T.T. Mottram, Quantitative analysis of the response of an electrochemical biosensor for progesterone in milk, Biosens. Bioelectron. 20 (2005) 2061-2070.

[5] R.L. Scipioni, R.H. Foote, An electronic probe versus milk progesterone as aids for reprodutive management of small dairy herds, J. Dairy Sci. 82 (1999) 1742-1745.

[6] R. Bobowiec, T. Studzinski, A. Babiarz, Thermoregulatory effects and electrical conductivity in vagina of cow during oestrus cycle, Arch. Exp. Veterinarmed. 4 (44) (1990) 573-579.

[7] B.L. Kyle, A.D. Kennedy, J.A. Small, Measurement of vaginal temperature by radiotelemetry for the prediction of estrus in beef cows, Theriogenology 49 (1998) 1437-1449.

[8] R. Herman, An introduction to electrical resistivity in geophysics, Am. J. Phys. 69 (2001) 943-952.

[9] A. Valente, R. Morais, C. Couto, J.H. Correia, Modeling, simulation and testing of a silicon soil moisture sensor based on the dual-probe heat-pulse method, Sens. Actuators A 115 (2004) 434-439.

[10] P.L. Senger, The estrus detection problem: new concepts, technologies, and possibilities, J. Dairy Sci. 77 (1994) 2745-2753.

[11] D.A. Schoeller, R.F. Kushner, Determination of body fluids by the impedance technique, IEEE Eng. Med. Biol. Mag. 8 (1998) 1921.

[12] A. I. Cano, Contributions to the measurement of electrical impedance for living tissue ischemia injury monitoring, PhD Thesis, Universitat Politècnica de Catalunya, Departament D'Enginyeria Electrònica, 2005.
[13] R.N. Baumgarter, Electrical impedance and total body electrical conductivity, in: A.F. Roche, S.B. Heymsfield, T.G. Lohman (Eds.), Human Body Composition, Human Kinectics, Champaign, IL, 1996, pp. 79-107.

[14] R. Morais, A. Valente, C. Couto, J.H. Correia, A wireless RF CMOS mixedsignal interface for soil moisture measurements, Sensors and Actuators A 115 (2004) 376-384.

\section{Biographies}

Raul Morais graduated in electrical engineering from the University of Trásos-Montes and Alto Douro (UTAD), Portugal in 1993. He obtained the MSc degree in industrial electronics in 1998 from the University of Minho, Portugal and the $\mathrm{PhD}$ degree in microelectronics in 2004 from the UTAD. Presently, he is an assistant professor in the Department of Electrical Engineering, UTAD. He is also a researcher in the signal processing and biotelemetry group at the Center of Technological Studies for Environment and Life (CETAV) of UTAD, and he is involved in the development of instrumentation solutions and mixed-signal sensing interfaces for agricultural applications.

António Luís G. Valente graduated in Electrical Engineering from the University of Trás-os-Montes and Alto Douro (UTAD), Portugal in 1994. He obtained the MSc degree in industrial electronics in 1999 from the University of Minho, Portugal and the PhD degree in microelectronics in 2004 from the UTAD. Presently, he is an assistant professor in the Department of Electrical Engineering, UTAD. He is also a researcher in the signal processing and biotelemetry group at the Center of Technological Studies for Environment and Life (CETAV) of UTAD and he is involved in the research of silicon microsensors for agriculture.

José Carlos Almeida graduated in animal science from the University of Trásos-Montes and Alto Douro (UTAD), Portugal in 1987. He obtained the PhD degree in animal reproduction in 2000 from UTAD. Presently, he is an assistant professor in Department of Animal Science, UTAD. He is also a researcher, at the Center of Animal and Veterinary Science (CECAV-UTAD), and his area of interest is reproduction in farm animals.

Amélia M. Silva graduated in biochemistry at the Faculty of Science and Technology, Coimbra University (FCTUC), Portugal in 1992. She received her MSc degree in cell biology from the FCTUC, Portugal in 1995 and her PhD in biochemistry (cellular biophysics speciality) from FCTUC, Portugal, in 2003. At the present, Amélia M. Silva, is an assistant professor at the Biological and Environmental Engineering Department, UTAD, Portugal. She is also a researcher at the Center of Technological Studies for Environment and Life (CETAV, at UTAD) and has been involved on cell physiology, biophysics and biochemistry studies on hormone secreting cells.

Salviano F.S.P. Soares graduated in electrical engineering from the University of Trás-os-Montes e Alto Douro (UTAD), Portugal in 1991. He obtained the MSc degree in electronics and telecomunications in 1995 from University of Aveiro (UA), Portugal, and the PhD degree in electrical engineering in 2003 from UA. Presently, he is an assistant professor in Department of Electrical Engineering, $\mathrm{UTAD}$, and also a researcher in the signal processing and biotelemetry group at the Center of Technological Studies for Environment and Life (CETAV) of UTAD. His main interest area is digital signal processing.

Manuel J. Cabral S. Reis received the $\mathrm{PhD}$ degree in electrical engineering and the MSc degree in electronics and telecommunications from the University of Aveiro, Portugal. Currently he is assistant professor in the Department of Engineering of University of Trás-os-Montes e Alto Douro (UTAD), Portugal. $\mathrm{He}$ is also a researcher at the Center of Technological Studies for Environment and Life (CETAV) of UTAD, where he is the director of signal processing and biotelemetry group. His research interests are in the area of signal processing, and include modelling and approximation, and problems such as sampling, interpolation, and signal reconstruction.

Ramiro Valentim graduated in zootechnic engineering from the University of Trás-os-Montes and Alto Douro (UTAD), Portugal in 1989. He obtained 
the MSc degree in animal science from IAMZ-CIHEAM (Mediterranean Agronomic Institute of Zaragoza - member of the Internacional Centre for Advanced Mediterranean Agronomic Studies) in 1994, Spain and the $\mathrm{PhD}$ degree in animal science in 2004 from UTAD. Presently, he is an assistant professor in Department of Zootechnic, ESA-IPB (Agrarian Superior School, Politechnic Institute of Bragança), Portugal. He is involved in the study of how environmental factor affects reproduction of local sheep and goat breeds.
Jorge Manuel Teixeira de Azevedo graduated in animal science from the University of Trás-os-Montes and Alto Douro (UTAD), Portugal in 1981. He obtained the $\mathrm{PhD}$ degree in animal science in 1994 from UTAD. Presently, he is a full professor in Department of Animal Science, UTAD, Portugal. He is also a researcher, at the Center of Animal and Veterinary Science (CECAV-UTAD), and his area of interest is animal production. 